\title{
Transhumanist Immortality: Understanding the Dream as a Nightmare
}

\author{
PABLO GARCÍA-BARRANQUERO \\ Universidad de Málaga \\ pablogarcia@uma.es \\ ORCID: 0000-0001-6472-5703
}

\begin{abstract}
This paper offers new arguments to reject the alleged dream of immortality. In order to do this, I firstly introduce an amendment to Michael Hauskeller's approach of the "immortalist fallacy". I argue that the conclusion "we (normally) do not want to live forever" does not follow from the premise "we (normally) do not want to die". Next, I propose the philosophical turn from "normally" to "under these circumstances" to resolve this logical error. Then, I review strong philosophical critiques of this transhumanist purpose of immortality in the literature. There are two key questions related to the possibility of fulfilling this goal: the hard problem of consciousness and the personal identity dilemma. Finally, I defend a specific type of indefinite life and justify that it is more desirable than our current limited life.
\end{abstract}

Keywords: Immortalist fallacy; immortality; indefinite life; transhumanism.

\section{Introduction}

\section{An optimistic vision of future technology}

Let me confess an almost daily concern: I do not want to die today. I am confident that I will not want to die tomorrow either. My anxiety about dying is based on my fear of the moment in which my conscious life will 
end. I cannot imagine what it would be like if I ceased to exist. This fear is an emotion which invades my mind and I can hardly suppress. Once dead, I will not be able to enjoy admiring the sunset in Oriental Bay, reading The Divine Comedy, or watching Blade Runner 2049 again. This concern is not mine alone: it drives the philosophy of existentialists, and much of both Eastern and Western religious thought. ${ }^{1}$ My anxiety may have deep-seated evolutionary origins, and it even seems to be shared by other animals. Will there ever be a solution to this common fear?

The advancement of science and technology in the past half-century is opening up unprecedented horizons. The world is transforming, everything is changing-not only in terms of the way we live, but also in terms of how long. With science and technology increasingly pushing back the frontier of death, is it possible that the "death of death" will be fulfilled in the distant future? (Wood 2016). There is a distinguished group of thinkers who proclaim the advent of the Singularity, where the evolution of Homo sapiens will be directed by ourselves. The Singularity refers to the point in time when all the advances in science and technology will cause unimaginable biological, cultural, and social changes, impossible to predict or understand before this event. In the Singularity, there will be no distinction between humans and machines, or between physical and virtual world. Raymond Kurzweil (2005, Ch. 1) suggests that we think about the way in which technology has evolved over the past 100 years and project it into the future: there will be an exponential growth of diverse forms of technological progress after which the meaning of human life will be radically different. In this future, we will conquer the fear of an inevitable death by eliminating the inevitability of death itself. Many of the defenders of the Singularity typically endorse some version of transhumanism $(\mathrm{H}+)$.

I do not deny that this debate would be radically different for an atheist compared to someone who believes in an afterlife. For the former, death is the end of our life. For the latter, death is only the end of our biological and earthly life before entering another plane of existence. My arguments hold for any rational person, regardless of their spiritual beliefs or lack thereof. 
$\mathrm{H}+$ is a philosophical, scientific-technological, and social way of thinking about the transformation of human beings. It provides opportunities by developing emerging technologies to extraordinarily enhance ourselves. ${ }^{2}$ $\mathrm{H}+$ will enable our society to be healthier, longer-lived, and smarter, capable of selecting from a wide range of enhanced attributes and new traits. Nick Bostrom (2008) or Anders Sandberg (2014), among others, present possible advantages and disadvantages-that is to say, benefits and risks-of these emerging technologies that could overcome traditional conditions in cognition, longevity, or physical performance. Their premise is that human beings at present do not enjoy the best of all possible states of existence. Thus, the human condition is questioned, it is no longer sacrosanct as some essentialists have defended (Kass 2003; Sandel 2007). On the basis of their optimistic vision of future technology, they want to free human beings from our biological chains, including death. Transhumanists justify this vision based on the assurance that technology is growing faster than we could have ever imagined, and it offers a new range of infinite possibilities and powers. In other words, today's science-fiction is a glimpse at tomorrow's reality. But what kind of world does technology have in store for us?

\section{Digital immortality: The transhumanist dream}

In a philosophical paper on life extension, Larry Temkin (2008) wonders whether living longer is inherently living better. In this work, I begin with the claim that I want to live longer, regardless of whether that means living better than I do now. This starting point raises a significantly different question: "Does wanting to live longer entail wanting to live forever?" (Agar 2010, Ch. 6; Gems 2003; Williams 1973).

From the perspective of $\mathrm{H}^{+}$, living longer is the first step towards the ultimate goal of conquering death. This perspective envisions no limits to humanity's ability for continuous enhancement. Furthermore, the de-

2 There is an extensive academic literature about the ideas defended by transhumanists in which the arguments for and against are exhaustively reviewed (Agar 2010; Diéguez 2017). See more briefly, Asla (2019); Hauskeller (2019). 
struction of the biosphere will not be an obstacle to our survival, if the form of immortality that the Singularity offers is not biological. For some transhumanists, while the body is simply “jelly” (Moravec 1989, 117), our minds may be uploaded into a computer (which is eternally functional) and, in this way, achieve digital immortality. Transhumanists claim that our hardware, like the human body, will be disposable. What will remain in perpetuity is our software, even enhanced by future technology. We will be able to transfer our ideas or memories into virtual world in which we will live better than we live now. David J. Chalmers explains (destructive) mind uploading as follows:

One possible form involves serial sectioning. Here one freezes a brain, and proceeds to analyze its structure layer-by-layer. In each layer one records the distribution of neurons and other relevant components, along with the character of their interconnections. One then loads all this information into a computer model that includes an accurate simulation of neural behavior and dynamics. The result might be an emulation of the original brain $(2010,42)$.

$\mathrm{H}+$ (implicitly) defends (destructive) mind uploading because the biological chains are entirety gone by this point. The idea of digital immortality is a form of life extension in which we would live forever and never die. The result might look like an avatar behaving, thinking, and reacting like a person on the basis of that person's digital archive. In this line, Kurzweil (2005, Ch. 7) considers that digital immortality is forever as long as someone takes care of the information. In a very strict sense, even this immortality would not be absolute. Some may claim that this transhumanist purpose of immortality would never be possible. Imagine that our digital selves are erased by a computer virus or the heat-death of the universe. However, I approach digital immortality as if it were a possibility for complete immortality: life without an end, with "life" no longer being tied to biological limitations. In contrast to digital immortality, indefinite life is a form of life extension in which humans have already cured aging but they can die from external causes, such as accidents, dehydration, or murder, among other possibilities (Glannon 2002, 343-344). Life is (potentially) forever, in biological terms. 
Even though life extension technology offers us the possibility to live and longer, even forever, we would remain vulnerable to the possibility for life to end, as "life" is tethered to biology. ${ }^{3}$

Immortality is a hypothetical scenario for which imagination is necessary because of the absolute lack of scientific-technological evidence. Accordingly, immortality is neither an expected nor a realistic result of treating aging. What is more, an extreme longevity, similar to Methuselah's life, does not imply that one can live forever. The goal of the rest of this paper is to reject the alleged dream of immortality. My structure will be as follows: in section 2, I will introduce an amendment to the immortalist fallacy (IF), inspired by Michael Hauskeller's approach (2013). In section 3, I will review strong philosophical critiques of this transhumanist purpose of immortality in the literature: the hard problem of consciousness and the personal identity dilemma. Finally, in section 4, I will conclude the paper by suggesting a specific type of indefinite life derived from section 1 and justified by section 2 that it could be more desirable than our current limited life. Thus, I will argue that this does not only change our concept of "death", but also our concept of "life".

\section{The immortalist fallacy: An amendment to Hauskeller's approach}

\subsection{A logical error?}

Everyone wants to enjoy as much time as possible with their loved ones, as long as their cognitive and physical capacities are tolerable, broadly speaking.

3 Nicholas Agar makes this distinction between a zero and non-zero immortality: “There's actually a big difference between immortality and negligible senescence. Whereas a negligible senescence being is likely to have a longer life span than a senescing one, an immortal being is guaranteed to. Immortal beings have a zero probability of dying over any future period of time. Negligible senescent beings have, in contrast, a nonzero probability of dying with each year that passes. The difference between them and us as we are now is that this probability does not increase" $(2010,113)$. Broadly speaking, we refer to the same distinction. However, I prefer to call it "indefinite life" because I argue that death is possible beyond the cure of aging. 
Many of us want more time for other purposes as well, like reading classic novels, visiting exotic countries, and watching cult movies, among other things. From this perspective, living longer could be better for humans. ${ }^{4}$ Many transhumanists doubt that there is anyone who rejects the dream of immortality (Bostrom 2008). Now, I discuss that most radical view of the life extension possibilities: living forever and never die.

Transhumanists argue that any significant extension of life we would turn us into better humans, in the sense that we would be better off than we are now. They assume that we appreciate being alive (life is good) and that we do not want to die (death is bad). Bostrom uses these premises to construct his main argument: (1) people "normally" appreciate being alive; (2) accordingly, they "normally" desire not to die; (3) they also have an (implicit) desire to have their life spans indefinitely extended; and (4) thus, they normally desire to live (potentially) forever (2008, 113-118). Bernard Williams, in his famous seminar chapter on immortality, stated a similar conclusion: "[W]anting something itself gives one a reason for avoiding death" (2000: 59). I assume a moderate position in this debate, but in order to establish it I first have to answer these two questions: (1) "Do we never want to die?”; and (2) “Do we want to live forever?”. A useful starting point is Hauskeller's approach (2013), which argues that Bostrom (2008) commits what the calls the IF in making his four-step arguments:

[T]his argument, plausible as it may seem at first sight, is misleading, because it is based on the premise that we (normally) do not want to die, which is then taken to imply that we do want live forever (i.e. have an implicit desire for indefinite life extension). Yet this conclusion is in fact not warranted. If you ask people whether they want to die, most of them will indeed deny it. However, if you as, the same people whether they want to go on living forever, you may find that most will deny this too (...). It is quite possible that a person does not want to die and still does not want to live forever. On the face of it his seems

4 In this paper, I assume a welfarist concept of living better. This concept entails several changes in anthropology, morality, or psychology, which "increase the chances of leading a good life in the relevant set of circumstances" (Savulescu et al. 2011, 16). I do not believe that the meaning of life can be considered by religious or traditional doctrines. 
to be a blatant contradiction. How can we at the same time not want to die and not want to live forever? Obviously, if you do not die you will live forever and the only way to avoid living forever is to die $(2013,89-90)$.

Hauskeller uses his critique of Bostrom's argument to reject the alleged dream of immortality. He argues that there is no logical entailment between the claim that "people (normally) do not want to die" and "people (normally) want to live forever". In my view, the key to dismantling Bostrom's argument is the qualifier of the premise: "normally". My position does not only serve as an amendment to Hauskeller's approach, but also as a distinct framework to reject the dream of immortality of transhumanists.

That a person does not "normally" want to die does not imply that the same person does not ever want to die (i.e. in the future). To clarify Hauskeller's approach, I propose a philosophical turn from "normally" to "under these circumstances". What does "normal" mean for Bostrom or Hauskeller in the IF? They do not clarify whether it is "ideally" "generally", "most of the time", or "most people". There are many possible meanings which would substantially change the argument. I understand "under these circumstances" as the anthropological, historical, psychological, and social context according to which every individual makes a claim about the IF. I do not want to die "under these circumstances" perhaps something that we all think by living a limited life now implies an implicit desire to live forever under these circumstances. This implicit desire to live forever is conditioned by two reasons: (1) I want to be alive today; (2) and I do not want to die today. I respond to the IF in my present. I cannot know what I would say in my future under other circumstances. ${ }^{5}$ The fear of death justifies my desire to live indefinitely, more than anything else.

Hauskeller thinks about the IF as a mortal human being, like any of us. Moreover, Bostrom and Kurzweil remain human beings, beyond their optimistic vision of future technology. They remain locked in the chains

With other words, I can say that it is incoherent to have any opinion about the attractiveness of living forever, or even of living to 1000 , or only 100 , because it is axiomatic that whether one wants to die now (or soon) depends on one's perceived quality of life at the time and in the subsequent future. I owe this comment to Aubrey de Grey. 
of biology. Death is the "inevitable destiny" of transhumanists if their dream does not come true. All of us are in the same scenario and we all project a future world that spans beyond our current limited life. The substantial difference between us is the new world that we want now. Thus, this reflection depends strongly on the context of every individual. I take a metaphysical step from Hauskeller's approach since I consider two possible scenarios in the IF: (a) the people being described are mortal; (b) the people being described are already immortal. For me, Hauskeller's question: "How can we at the same time not want to die and not want to live forever?" $(2013,90)$ should be substituted by the statement: "We can under different circumstances not want to die and not want to live forever". I propose dissolving the contradiction by showing different responses in these opposing scenarios. We can desire or reject the dream of immortality, but we do it under current circumstances, according to which our life is limited and death is possible.

In the first scenario, in which we are mortals, we are delaying the hands of the clock. Time is against us and death will come eventually. In the second scenario, in which we are already immortal, we would live without having to look at the clock. The clock does not exist anymore. In the first scenario, death is the "inevitable destiny" because mortal beings are all condemned to die, no matter how long we are able to delay our end. In the second scenario, death could be a "salvation" if it were the only way to move beyond our temporal existence. Otherwise, life would be a prison from which we could never escape. According to Hauskeller's approach, death would not always be something bad.

\subsection{Is death always bad?}

I have amended Hauskeller's approach to the IF to suggest that where he qualifies his claims with "normally", it would be better to say "under these circumstances". Now, I evaluate the concept of "death" from my previous division between mortals and immortals. I follow Hauskeller's approach. As he says: 
Yet even if we should decide that death is indeed an evil, non-death (or living forever) need not be a good. From the fact that a person does not want to die it follows neither that (a) death is an evil to them, nor that (b) living forever is a good to them. If this were a valid argument, which it is not, then we could just as easily conclude from the fact that a person does not want to live forever, that, to them, (a) life is an evil and (b) dying is a good $(2013,99)$.

Like Hauskeller, I strongly argue that the desire to live forever does not the imply desire not to die. The philosophical turn from "normally" to "under these circumstances" also shows how death could be "bad" or "good" depending on the context of every individual. For many people, the case a persistent vegetative state is an example of a situation in which death might be considered a "salvation" rather than an "inevitable destiny". There is nothing in the desire not to die that infers that living forever is an inherently desire. The question is conditioned by the type of life that we would live. ${ }^{6}$ Following Hauskeller's approach, but incorporating my amendment, I present the concept of death in the IF:

1. That a person does not want to die does not imply that either (a) death is bad to this person, or that (b) living forever is a good to this person. That a person does not want to die implies that this person wants to avoid it under a specific set of circumstances. That a person does not want to die implies that this person to be alive under these circumstances, but not that this person wishes to be immortal.

2. That a person does not want to live forever does not imply that either (a) life is bad to this person, or that (b) death is a good to this person. That a person does not want to live forever implies that this person wants to die under a specific set of circumstances. That a person does not want to live forever implies that this person to be alive today, but not forever.

6 In the previous case the decisive reason was health. There are examples where the most influential factors are the culture and time. Hara-kiri, is the classic Japanese ritual suicide by disembowelment. Samurai voluntarily stuck a dagger into their stomachs so as not to fall into the hands of the enemy or to atone for dishonor, or for a failure. 
Death is not essentially "bad" or "good" "undesirable" or "desirable"and either is life. I have proposed that whether death is seen as an "inevitable destiny" or as a "salvation" depends entirely on the circumstances of a person's life. Someone who is happy with the good experiences that life can provide may have compelling reasons to want to keep having those experiences indefinitely-or at least until they no longer seem so good.

However, someone could say that death could be avoided is only if we wished to (there would always be a way out). In this vein, I will explain what I call the obligatory nature of immortality in $\mathrm{H}+$. Taking this as a baseline, I will defend my own concept of death related to the IF. Similarly, I will present my concept of "life" according to this obligatory nature of immortality in section 4 . First, the Singularity is the point in time at which all the advances in technology will change the world as we know it today. This event will affect all humans without exception (Kurzweil 2005, Ch. 1). In other words, transhumanists seem to assume that all these changes will be universally accepted. Second, digital immortality through that the (destructive) mind uploading would guarantee one could live forever and never die. Would technological suicide be possible? For these transhumanists, it is rather strange to end life when we have conquered death technologically after centuries. Kurzweil says that if we take care of the information, we will never die. Could we totally cease to exist after our upload? Someone could also consider that this possibility implies that the only true form of immortality would be an indefinite life. According to the above, I justify what I have introduced previously in relation to the IF: (1) death is the "inevitable destiny" in our current limited life; and (2) death would be the "salvation" in immortality only if it were possible. And transhumanists seem to disagree with one scenario in which death could be optional.

I have started my discussion with this question: "Do you never want to die?". Rather, we should ask: "Do you never want to die under the current circumstances?”. Along this line, John Harris says: “Most people fear death, and the prospect of personal extended life-span is likely to be welcomed" (2000: 59). Absolutely. Transhumanists promise to live forever because they know that the fear of death would entail the acceptance of any type 
of life. Anything would be better than nothing for them. These authors consider that the IF to be meaningless, and conceptualize only a single scenario in which they conquer death and live forever. Thereby, I have already presented a possible solution to the first question at the beginning of this section: “Do we not want to die?". No. Broadly speaking, and under tolerable circumstances, today we want to be alive. Tomorrow life itself might show us a world that we may prefer to escape through death. One of the most difficulties is to predict how events in our lives will unfold from one day to the next.

Having shown the logical error from the perspective of individual desire, I take one metaphysical step. If wanting to live forever were our desire, would (destructive) mind uploading be possible? Would future technology solve all the challenges of digital immortality?

\section{Serious arguments to reject digital immortality}

\subsection{Strong philosophical critiques}

I have presented my amendment to Hauskeller's approach to the IF (section 2). Now, I consider it useful to review the critiques that of the dream of immortality that are raised in the literature. Transhumanists have already shown all the (possible) benefits that (destructive) mind uploading would have-and how it would improve people's lives in all their aspects. For instance: (1) people could create multiple avatars of themselves to accomplish their goals; (2) people could enjoy unimaginable pleasures for our senses; or (3) people could save an emergency copy of their profiles. However, I point out different problems that this type of "life" could pose for us in the distant future. Everything is speculative, but I also think a scenario not as desirable as the one proposed by transhumanists. From this perspective, it is not only about the individual desire to want to live forever but also about whether this desire can be fulfilled technologically. Transhumanists imagine what they would like to happen. Where there is a will, there is (not always) a way. Perhaps this (great) difficulty is what really concerns 
transhumanists. Hence, I argue that there are strong reasons to show that the transhumanist goal is not an easy one.

By "strong" critiques, I understand those that directly undermine the very concept of digital immortality. Not surprisingly, there are serious difficulties in meeting this goal from a technological perspective. Here, I follow the ideas of Chalmers (2010) who argues that there are two key issues in the (destructive) mind uploading debate: the hard problem of consciousness and the personal identity dilemma. Therefore, my rejection of digital immortality is related to these two questions: (1) "Will an uploaded version of me be conscious?"; and (2) "Will I, upon uploading, still be me?”. The concept of mind that Bostrom (2008) or Kurzweil (2005) defend is quite widely discussed in neuroscience and philosophy. They assume that the mind is something analogous to software, to an information pattern that can be transferred to different hardware and function correctly. Consciousness would be one of those functions, such as the ability of my laptop to monitor its own functionality, to distinguish self from non-self by identifying computer viruses, and to understand voice commands. However, many thinkers show that this is a reduced version of who we are and that we hardly know what consciousness is-besides, we are not certain at all that it is everything that makes us who we are. Even supposing that consciousness were not a problem, the personal identity dilemma would still be present. For instance, if I could make copies of my mind, or I could transfer it to a silicon device, nothing would guarantee me that "he", "she", or "that" is myself (Hopkins 2012; Pigliucci 2014). Agar shows this dilemma in relation to different possible scenarios after the uploading. In short:

There are two possible consequences of uploading. The advocates of strong AI think that the computers we are uploaded into are capable of conscious thought. If Kurzweil is right, you will not only survive, but your powers of thought will be radically enhanced. If the doubters are right, then uploading is a nothing more than a novel way to commit suicide $(2010,63)$.

We would try to avoid death by uploading our minds into a machine, but we could die in the process. If this is the case, then (destructive) mind 
uploading would just be a sophisticated way to end our lives. Even more, I suggest that we are afraid of death but we are not aware that we could face an even more fearful future. The most frightening monsters are not dragons or witches; they are those that we cannot yet imagine. There is a third possible scenario besides mind uploading way to end our lives or the transhumanist success: we have survived but neither us nor the world around us is what we really wanted. In this vein, João Pedro de Magalhães proposes a similar idea when he says that:

Due to the both creative and destructive nature of the human mind, the dilemma is whether the technological singularity will be a bridge to wonderland or if it will mean the end of human civilization $(2004,85)$.

This nightmare of immortality characterizes the possible scenario triggered resulting from $\mathrm{H}+$ in which we would be obliged to accept not only all the technological changes of the Singularity, but also the impossibility of ceasing to exist. After the Singularity, the type of life that we would live would be radically different from our current ones, and according to $\mathrm{H}^{+}$, these new lives would be much better than anything that we can dream of. Now, we imagine our lives projected towards a vague, abstract idea of finality, knowing we are mortal (cf. Kitcher 2014, 99-100). A serious for everyone to consider would be: "Do you know what mind uploading would be to say yes to the transhumanist dream?".

Typically, only the two extreme options of the debate are presented for consideration by the wider society: essentialist mortality (Kass 2003) and digital immortality (Kurzweil 2005). However, it is possible that most people unknowingly hold a moderate position like the one that Hauskeller and I have described previously. Based on my amendment to the IF, they would want to keep living indefinitely as long as they can live under their current circumstances. They would not want to live forever because they can imagine themselves under other circumstances in which they would rather die. The probability of seeing paradise on Earth is the same as that of seeing hell. What is more, they are not two sides of a coin since we do not even know whether there are two or more options. 
Hauskeller $(2013,100)$ thinks that many people want to live forever simply because they imagine a radically different future. From my perspective, there is an assumption that in the future we will live better than now. $\mathrm{H}+$ seems to consider only the success of (destructive) mind uploading, but it is also possible that life in the future could be more unbearable anything we could ever imagine. I would like to live a life in which I recognized myself. Kurzweil (2005) states that life will be better than now after the Singularity. We will have a brain and body beyond the limits of biology. He wants our existence to be as we need it and want it at any time. We will be the ones to create ourselves. Some human beings would want to have a different avatar every day; others would reject being able to imagine that they are angels and that they were born with feathered wings on their backs. Transhumanists want immortality, but they are mortal. They, and all of us, will be released from their biological chains after the explosion of the Singularity. They just need to be patient. ${ }^{7}$ However, what type of life we would live without death?

\section{The defense of a specific type of indefinite life}

\subsection{Changing the concepts of "death" and "life"}

In section 3, I have presented strong critiques to reject the dream of immortality. Now, I will defend my own approach. One of the question which started my argumentation was: “Do you want to live forever?”. In the literature, this question has also appeared as follows: "Is more life always better?" (Gems 2003).

I say that it depends on many factors. Following my argument that the desire for ongoing life is context-dependent, most people will want to keep living if the set of circumstances is ideal for them; for instance, if they can continue to live with their loves ones, and under cognitively and

The transhumanist perspective actually claims to want this vision of the future, rather than merely starting that "this is the inevitable future towards which we are headed". The prediction of a certain future does not necessarily imply that this is what one wants. For many others, there is a very large gap between the future that may lie ahead and what they would really want. 
physically tolerable conditions. Upon assessing my current circumstances, today I would answer: yes, more life is indeed better. Nevertheless, I am sure that there could be a time when my circumstances change so much that I would not want to continue to endure them. If a digital immortal existence were ultimately boring or oppressive, I might desire to end it rather than endure it forever. I defend that the answer to the question will always be both personal and temporary. And it is difficult, if not impossible, for the person who wants to live to understand the perspective of a person for whom death is desirable. At the same time, no one knows for sure whether they will ever change their desire to live, or to die. The only question that I can possible answer is whether I myself would prefer to keep living rather die under the current circumstances. From my view, absolutely. I do not want to die today. I probably will not want to die tomorrow either. The fear of death is present in my current limited life. Does this imply my desire to live forever? Not really, as I will explain below.

The question is not only about the value of changing the concept of "death" but also the concept of "life". Not being able to die, one of the greatest milestones that humans dream of, implies a radical modification of what we understand by our existence as humans. $\mathrm{H}+$ promises to evade the "inevitable destiny" of death through an optimistic vision of future technology. However, what would be the price? According to my amendment to Hauskeller's approach, we think about overcoming death from our finitude, but we also project the type of life we would like to live in the future. Now, if we were immortal would we want a similar life to the current limited one, in terms of our interests and values? It seems that, in one way or another, we would like to prolong a state of joy to infinity. We do not want what we do not know, what is beyond the limits of our mind, although this may be better. Our concept of "life" depends on who we are-it is the view from where we stand, so to speak. In other words, if we were not Homo sapiens, or even something similar, it is entirely possible that the concept of "life" would not exist-or at least, not in its current form. Enjoying certain activities or dreaming of a better future is specifically human. One question this raise is whether as a transhuman we would retain the same desire for immortality 
that we have as humans. $\mathrm{H}+$ takes one metaphysical step further by detaching itself from the biological body. If we cannot achieve the desired digital immortality, there is no reason to be uploaded. We would like to admire the sunset at Oriental Bay, read The Divine Comedy, or, watch Blade Runner 2049 once again. In this dystopian movie, Ryan Gosling walks through a desolate and demolished planet Earth where all our omens have come true. However, this very pessimistic future is imaginable for us. Thus far, we project a life that is similar to the one that we live today. All of these activities would be possible in a virtual world as long as the upload is a success of $\mathrm{H}+$.

I argue that it is impossible to know whether I would carry out all of these activities in a way that is similar to what I want under current circumstances. I would like to keep living if and only if the type of life that I would lead were not radically different from the current limited life that I enjoy today. Now, would I want my mind to be in a virtual world or something like that? Kurzweil (2005, Ch. 7) considers that the meaning of life is to appreciate and create a kind of knowledge which improves itself to direct us towards a higher "order". Therefore, death is a tragedy if a person's information is lost in time. True immortality would only be possible if we uploaded our mind into a computer. I do not claim that meaning of the life as envisioned by $\mathrm{H}+$ is the desire of all humans. Where they say "we", they should say "I", or "my colleagues".

I defend a change in the concepts of "death" and "life" as follows: if X is death and $\mathrm{Y}$ is life, humans only worry about $\mathrm{X}$ without thinking that $\mathrm{Y}$ changes when $\mathrm{X}$ disappears. The interdependence between $\mathrm{X}$ and $\mathrm{Y}$ is so strong that we cannot imagine a world without $\mathrm{X}$ in which $\mathrm{Y}$ is still $\mathrm{Y}$. A world without $X$ in which $Y$ is still $Y$ is impossible because $X$ does not exist anymore. Therefore, a world without $X$ forces us to think of a world where Y becomes Y'. Transhumanists promise us a world without $X$ in which Y' will be radically different from Y. From the TH perspective, we should not fear Y' for two reasons: (1) we would accept $Y^{\prime}$ since it is the consequence of the conquest of $\mathrm{X}$; and (2) we would accept that $\mathrm{Y}^{\prime}$ is not simply the consequence of the conquest of $\mathrm{X}$, but also the possibility of living better than our current limited Y. However, if Y' does not practically resemble $\mathrm{Y}$ at 
all, is it worth conquering $\mathrm{X}$ ? Most of society might want $\mathrm{Y}$ without $\mathrm{X}$, but is it known that Y' will in no way resemble our Y? Is it possible to imagine a life without death? Answering David Gems' question, one could say that a longer life is not always a better life.

\subsection{What do I want now?}

Now, let me confess what my dream is. I imagine a life free of aging and other diseases that limit my activities, plans, projects, and time with my loved ones; a life in which I would (potentially) live forever because I want to keep living indefinitely. However, I cannot give an answer as to how many years I would like to live. 10 more years? 100 more years? Maybe, I would say, a little more for now. There are open horizons of possibilities which I would still like to explore. If you ask me in a distant future, in which the transhumanist purpose has come true, my answer would surely be different. I am afraid to close my eyes and not be able to open them once again, but what if, in a dystopian scenario, I was afraid of never being able to close them and always having to continue looking at a terrifying world? I imagine that nobody finds it desirable to die for what is known as external causes: accidents, dehydration, or murder, among other possibilities. I, under current circumstances, would not want to die from any of the above. I convert into a virtue what for $\mathrm{H}+$ is a sign of weakness: the biological body. The fact of having it gives us the possibility to die both when we do not want and when we want to. One could desire life extension without desiring the immortality. On some occasions, one must sacrifice something to obtain something else.

Following my previous reflection, I fear X but I am aware that without $\mathrm{X}$, I could not keep Y, at least as I know Y today. I do not defend a static Y because my circumstances are changing day after day. It is a $Y$ recognized by me and those around me. I do not accept an unknown Y' just to avoid $\mathrm{X}$. What is more, I do not give up $\mathrm{X}$ to have an “eternal” $\mathrm{Y}$ '. Therefore, I do not accept what $\mathrm{H}+$ defends: the desirability of uploading our minds into a computer. First, this desire is quite improbable to achieve in the future. Second, if it were real, this type of existence implies something unknown 
that many people would not necessarily want. It is not, in other words, an inherent desirability.

This specific type of indefinite life that I think humans should seek lies between our current limited life and the dream of immortality: we would be assuming that it would be optional to want to keep living, that death would be avoidable for a certain time, and that it would be reversible, since we would have a "salvation". We could continue adding years of life, but, on the other hand, we would be unless there are external causes the ones who we decide if we want to die. It seems to me, that this might indeed be the central point of the argument: what does one want, to live forever? No. To die? Also no. What is desired is the ability to decide to end.

My current concern is not wanting to die. Death is the end of my life. My desire is not to face the moment in which I will lose everything. I do not want to live forever and I reject the transhumanist dream. How can I accept what I can hardly imagine? If it were possible, I would want to live better as long as my life had a very similar meaning as it has today. These are my conditions, my set of circumstances, under which I accept the idea of an unending life. Everything would change under other circumstances. This is what I have called the specific type of indefinite life.

I believe that I have answered the IF discussed by Hauskeller. Nevertheless, I need some more time to think about it.

\section{Concluding remarks}

In this paper, I have analyzed and discussed the transhumanist dream of immortality. First, I have suggested an amendment to Hauskeller's approach of the IF. The key to disambiguating the IF is the imprecise premise "normally". I propose a philosophical turn towards context-dependency, so that the desire to live is considered to be dependent on the circumstances of every individual. The value of death is related to these circumstances. Second, I have reviewed strong critiques against mind uploading in the literature. Two great challenges about mind uploading were presented by Chalmers (2010): the problem of consciousness and the personal identity dilemma. 
According to the above, digital immortality could be a nightmare for humans. Death would be a "salvation" when we do not want to live forever, if and only if this were possible. Otherwise, the immortality will no longer be so much of a dream. Finally, I have argued in favor of a specific indefinite life which is more desirable than our current limited life. I would like to enjoy a life in which I would recognize myself, my loved ones, and the world where I live. Would there be anything better than deciding when is a good time to die? Once those things are no longer possible, my current fear of death should fade into acceptance and I would be released from this fear.

\section{Acknowledgments}

I would like to thank Nicholas Agar, Suzanne Day, Aubrey de Grey, Antonio Diéguez, Walter Glannon, Alejandro González Jiménez-Peña, Eric Juengst, Alfredo Marcos, Andrés Moya, Andrés Ortigosa, and Jon Rueda, who read previous versions of this paper and made helpful suggestions. I owe Antonio Yuste-Ginel the idea of modifying the qualifier "normally". This idea was the catalyst for the rest of the paper. Additionally, I thank two anonymous reviewers of Scientia et Fides and the editor for their comments on earlier drafts. The research activity of Pablo García-Barranquero was supported by MECD-FPU 2014/02041 and Contrato Puente para Doctores de la Universidad de Málaga.

\section{References}

Agar, Nicholas. 2010. Humanity's End: Why We Should Reject Radical Enhancement. Cambridge, MA: MIT University Press.

Asla, Mariano. 2019. "On the limits, imperfections and evils of the human condition. Biological improvement from a thomistic perspective.” Scientia et Fides 7(2): 77-95. DOI: http://dx.doi.org/10.12775/SetF.2019.017

Bostrom, Nick. 2008. “Why I want to be a posthuman when I grow up.” In Medical Enhancement and Posthumanity, edited by Berth Gordijn., and Ruth Chadwick, 107-136. Dordrecht: Springer.

Chalmers, David J. 2010. "The singularity: A philosophical analysis." Journal of Consciousness Studies 17: 7-65. 
de Magalhães, João P. 2004. “Alice’s dilemma.” Futures 36(1): 85-89.

Diéguez, Antonio. 2017. Transhumanismo: La búsqueda tecnológica del mejoramiento humano. Barcelona: Herder Editorial.

Gems, David. 2003. "Is more life always better?: The new biology of aging and the meaning of life.” Hastings Center Report 33(4): 31-39.

Glannon, Walter. 2002. "Extending the human life span.” The Journal of Medicine and Philosophy 27(3): 339-354.

Harris, John. 2000. “Intimations of immortality.” Science 288(5463): 59.

Hauskeller, Michael. 2013. Better Humans?: Understanding the Enhancement Project. Durham, NC: Routledge.

Hauskeller, Michael. 2019. “Ephemeroi-human vulnerability, transhumanism, and the meaning of life.” Scientia et Fides 7(2): 9-21. DOI: http://dx.doi.org/10.12775/ SetF.2019.013

Hopkins, Patrick D. 2012. "Why uploading will not work, or, the ghosts haunting transhumanism.” International Journal of Machine Consciousness 4(01): 229-243.

Kass, Leon. 2003. Beyond Therapy: Biotechnology and the Pursuit of Happiness. New York: Harper Collins.

Kitcher, Philip. 2014. Life after Faith. The Case for Secular Humanism. New Haven: Yale University Press.

Kurzweil, Raymond. 2005. The Singularity Is Near: When Humans Transcend Biology. New York: Penguin.

Moravec, Hans. 1989. Mind Children. Harvard: Harvard University Press.

Pigliucci, Massimo. 2014. "Mind Uploading: A philosophical counter-analysis”. In Intelligence Unbound: Future of Uploaded and Machine Minds, edited by Russel Blackford., and Damien Broderick, 119-130. Oxford: Wiley-Blackwell.

Sandberg, Anders. 2014. “Transhumanism and the meaning of life.” In Religion and Transhumanism: The Unknown Future of Human Enhancement, edited by Tracy Trothen., and Calvin Mercer, 3-22. Oxford: Praeger.

Sandel, Michael J. 2007. The Case against Perfection. Harvard: Harvard University Press. Savulescu, Julian., Anders Sandberg, and Guy Kahane. 2011. "Enhancement and well-being.” In Enhancing Human Capacities, edited by Julian Savulescu., Ruud ter Meulen, and Guy Kahane, 3-18. Oxford: Wiley-Blackwell.

Temkin, Larry. 2008. “Is living longer living better?” Journal of Applied Philosophy, 25(3): 193-210.

Williams, Bernard. 1973. "The Makropoulos Case: Reflections on the Tedium of Immortality." Problems of the Self. Cambridge: Cambridge University Press.

Wood, David. 2016. The Abolition of Aging. The Forthcoming Radical Extension of Healthy Human Longevity. London: Delta Wisdom. 